\title{
La conducta financiera entre estudiantes universitarios emprendedores
}

\author{
Laura Angélica Décaro-Santiago ${ }^{1}$ \\ Universidad Autónoma del Estado de México \\ ladecaros@uaemex.mx \\ María Guadalupe Soriano-Hernández ${ }^{2}$ \\ Universidad Autónoma del Estado de México \\ mgsorianoh@uaemex.mx \\ Juan Pedro Benítez-Guadarrama ${ }^{3}$ \\ Universidad Autónoma del Estado de México \\ jpbenitezg@uaemex.mx \\ Juana Gabriela Soriano-Hernández ${ }^{4}$ \\ Universidad Autónoma del Estado de México \\ jgsorianoh@uaemex.mx
}

Fecha de recepción: 10 de marzo de 2020

Fecha de aprobación: 06 de noviembre de 2020

Fecha de publicación: 18 de marzo de 2021

Cómo citar este artículo / To reference this article / Comment citer cet article / Para citar este artigo:

Décaro-Santiago, L. A.; Soriano-Hernández, M. G.; Benítez-Guadarrama, J. P.; Soriano-Hernández, J. G. (2020). La conducta financiera entre estudiantes universitarios emprendedores. Revista Escuela de Administración de Negocios, (89), 51-68.

DOI: https://doi.org/10.21158/01208160.n89.2020.2816

\section{Resumen}

Para reducir parte de las barreras del emprendimiento y disminuir los índices de mortandad de las empresas de nueva creación, es necesario generar estrategias que fomenten decisiones en temas de finanzas personales entre los estudiantes universitarios, de manera más consciente y precisa. Teniendo en cuenta que el papel de las instituciones de educación superior es determinante para fortalecer dicha capacidad, el objetivo de esta investigación es explorar la conducta financiera de los estudiantes universitarios de dos centros universitarios de la Universidad Autónoma del Estado de México (UAEM). Esto al hacer la distinción entre aquellos que son emprendedores, emprendedores potenciales y aquellos que no los son, con la finalidad de identificar conductas en temas de crédito, ahorro y administración del dinero, sin olvidar los niveles de inclusión en el sistema financiero formal. La recolección de los datos se obtuvo a través de la aplicación de

1 Doctorado en Ciencias Administrativas por el Instituto de Estudios Universitarios de Puebla y Maestría en administración por la Universidad Autónoma del Estado de México. ORCID: https://orcid.org/0000-0002-6778-3359

2 Doctorado en Ciencias por el Colegio de Postgraduados y Maestría en Impuestos por Colegio de posgrado de la Ciudad de México. ORCID: https://orcid.org/0000-0001-5682-8155

3 Doctorado en Ciencias de los Fiscal por el Instituto Especializado para Ejecutivos AC y Maestría en impuestos por la Escuela Superior de Estudios Humanísticos. ORCID: https://orcid.org/0000-0002-2826-6359

4 Doctorado en derecho procesal penal por Instituto de Zumpango en Estudios Superiores y Maestría en Administración por la Universidad Autónoma del Estado de México. ORCID: https://orcid.org/0000-0002-3896-5647 
un cuestionario de preguntas cerradas. La muestra se conformó con 518 estudiantes de Administración, Contaduría, Derecho, Psicología, Ingeniería en Computación e Informática. Se utilizaron herramientas de medición tales como frecuencias y pruebas de asociación de variables de la estadística descriptiva. Entre los principales resultados se identifica que el 14,5\% de los estudiantes son emprendedores, mientras que el 69,7 $\%$ son emprendedores potenciales. Además, se identifica dependencia del estatus del emprendimiento con la inclusión al crédito formal, el pago de tarjetas de crédito, el pago a tiempo de las cuentas, el análisis del gasto hormiga, el registro de los gastos y la asignación de recursos.

Palabras clave: educación financiera; conducta financiera; emprendimiento; emprendimiento universitario; potencial emprendedor; inclusión financiera; administración del dinero.

\title{
Financial behavior among entrepreneurial university students
}

\begin{abstract}
In order to reduce some of the barriers to entrepreneurship and decrease the mortality rates of newly established enterprises, it is necessary to create strategies that encourage more conscious and accurate decisions on personal finance issues among university students. Considering that the role of higher education institutions is determinant to strengthen such capacity, the aim of this research is to explore the financial behavior of university students from two university centers of Universidad Autónoma del Estado de México (UAEM). This is performed by distinguishing between those who are entrepreneurs or potential entrepreneurs, and those who are not, in order to identify behaviors towards credit, savings, and money management issues, without forgetting the levels of inclusion in the formal financial system. Data collection took place through the application of a questionnaire with closed questions to a sample of 518 students of Administration, Accounting, Law, Psychology, Computer Engineering, and Computer Science. Measurement tools such as frequencies and tests for association of variables of descriptive statistics were used. Among the major results, we identified that $14.5 \%$ of the students are entrepreneurs, while $69.7 \%$ are potential entrepreneurs. In addition, we identified the dependence on the status of entrepreneurship including formal credit, payment of credit cards, timely payment of bills, analysis of ant-expenditure, recording of expenses, and allocation of resources.
\end{abstract}

Keywords: financial education; financial behavior; entrepreneurship; university entrepreneurship; entrepreneurial potential; financial inclusion; money management.

\section{Comportamento financeiro entre estudantes universitários empreendedores}

\section{Resumo}

Para diminuir parte das barreiras do empreendedorismo e diminuir as taxas de mortalidade de empresas recém-criadas, é necessário gerar estratégias que promovam decisões sobre temas de finanças pessoais entre os estudantes universitários, de forma mais consciente e precisa. Levando em consideração que o papel das instituições de ensino superior é decisivo para fortalecer essa capacidade, o objetivo desta pesquisa é explorar o comportamento financeiro dos estudantes universitários de dois centros universitários da Universidade Autônoma do Estado do México (UAEM). Isto fazendo a distinção entre empresários, empresários 
potenciais ou não, com a finalidade de identificar comportamentos em matéria de crédito, poupança e administração de dinheiro, sem esquecer os níveis de inclusão no sistema financeiro formal. A coleta de dados foi obtida através da aplicação de um questionário com perguntas fechadas. A amostra foi composta por 518 estudantes de Administração, Ciências Contábeis, Direito, Psicologia, Engenharia da Computação e Informática. Foram utilizadas ferramentas de mensuração como frequências e testes de associação de variáveis de estatísticas descritivas. Dentre os principais resultados, identifica-se que 14,5\% dos alunos são empreendedores, enquanto $69,7 \%$ são empreendedores potenciais. Além disso, a dependência da situação do empreendimento é identificada com a inclusão de crédito formal, pagamento de cartões de crédito, pagamento de contas em dia, análise de gastos, registro de gastos e atribuição de recursos.

Palavras-chave: educação financeira; conduta financeira; empreendedorismo; empreendedorismo universitário; empreendedor potencial, inclusão financeira; administração de dinheiro.

\section{Analyse des comportements financiers des étudiants de gestion d'entreprise}

\section{Résumé}

Pour réduire les barrières d'accès à l'entrepreneuriat et diminuer les taux de faillite des jeunes entreprises, il faut générer des stratégies renforçant les prises de décisions conscientes touchant aux finances personnelles des étudiants universitaires. Compte tenu du rôle déterminant des établissements d'enseignement supérieur pour renforcer cette capacité décisionnaire, cette investigation analysera le comportement financier des étudiants universitaires de deux sites de l'Université Autonome de l'État de México (UAEM). Cette investigation fera la distinction entre les étudiants de cursus de gestion d'entreprise, les étudiants étant de potentiels entrepreneurs, et ceux qui ne le sont pas, afin d'identifier les comportements en matière de crédit, d'épargne, de gestion financière et de taux d'utilisation du système financier institutionnel. La collecte des données a été mise en place grâce à un questionnaire fermé. L'échantillon, qui se compose de 518 étudiants de gestion, comptabilité, droit, psychologie et génie informatique, a été soumis à des mesures de fréquences et des tests d'association de variables statistiques et descriptives. Les principaux résultats montrent que $14,5 \%$ des étudiants sont des entrepreneurs, $69,7 \%$ sont des entrepreneurs potentiels, et que le statut d'entrepreneur dépend d'une bonne utilisation du crédit, du paiement ponctuel des cartes de crédit et des factures, de l'analyse précise des dépenses et de l'allocation stricte des ressources.

Mots-clés: étudiants universitaires; entrepreneuriat; entrepreneuriat universitaire; entrepreneurs potentiels; inclusion financière; éducation financière. 


\section{Introducción}

$\mathrm{D}$ esde que nuestra sociedad se encuentra estructurada bajo los conceptos del capitalismo, el libre mercado y la globalización, el emprendedor se han convertido en uno de los actores centrales impulsores de la economía, toda vez que son el primer eslabón en la gestión de nuevas empresas, dado que promueven la inversión, la formación de nuevos empleos, la generación de riqueza y un mayor número de opciones en el mercado para satisfacer las necesidades de los consumidores (Lupiáñez, Priede y López-Cózar, 2014; Salinas y Osorio 2012; Sparano, 2014). De esto se deriva la urgencia de los países por identificar, en primera instancia, las barreras que lo limitan; para luego, ejercer acciones para abatirlas, $y_{\text {, }}$ finalmente, fomentar emprendimientos con tintes de innovación.

Si bien respecto al primer punto diversos estudios han enlistado las barreras del emprendimiento, en cada una de ellas aparece el limitado acceso al financiamiento (GEM, 2013; IMCO, 2014; Hernández, González y Herrera, 2016; Messina y Pena, 2016). Sin entrar en detalle sobre el financiamiento destinado a emprender, simplemente en México, en general, es carente. Según datos de la Encuesta Nacional de Inclusión Financiera 2015 _ENIF_, solo el $29 \%$ de los adultos mexicanos tienen acceso al crédito formal (CNBV, 2017; Conaif, 2016;). Las razones, problemas en el buró de crédito, la falta de comprobación de ingresos o insuficiencia, no contar con los documentos y no tener historial crediticio (CNBV, 2017). Estos datos resultan relevantes, pues la población objeto de esta investigación son los estudiantes universitarios, quienes, desafortunadamente, forman parte de la población subatendida por las instituciones financieras, elemento que alimenta las barreras del emprendimiento.

¿Cuál es el interés de aterrizar la investigación en los estudiantes universitarios? Existe una fuerte exigencia por el emprendimiento innovador en la región de
América Latina y el Caribe; de manera más clara, los índices de emprendimiento de la región son favorables, sin embargo, la innovación tiene poca presencia, lo que compromete la competitividad genuina, sustentable y acumulativa (Alonso y Fracchia, 2011). En este sentido, los estudiantes universitarios de las diversas áreas tienen la capacidad de emprendery la posibilidad de hacerlo con innovación — aplicando y trasladando los conocimientos adquiridos-; por tanto, el papel de las IES resulta crucial. Evidencia de esto es la participación de universidades estadounidenses en la generación de patentes, lo que dio vida a 5171 start-up desde 1980, generando un incremento de ingresos de 160 millones de dólares a 1,4 mil millones de dólares de 1994 al 2005 (Olaya, Berbejal-Mirabent y Duarte, 2014).

Por tanto, las IES deben identificar y contribuir con acciones que ataquen las causas de las barreras del emprendimiento, las cuales, para el caso, se centran en el perfil financiero del estudiante universitario emprendedor. La directriz para las IES en este tema deberá ser el de fortalecer el perfil financiero del joven, reforzando con conocimientos y el uso consciente de estos en la obtención y el manejo de los recursos monetarios como medio para alcanzar los objetivos; esto, de manera correcta, alimentará los niveles de seguridad del estudiante al tomar decisiones de índole financiera. Esto en razón a que los bajos niveles de alfabetización financiera en los emprendedores dificultan la financiación en etapas tempranas de un proyecto (Atkinson, 2017).

Así, las conductas financieras se encuentran estrechamente relacionadas con el nivel de alfabetización financiera (Lusardi y Mitchell, 2016), cuyos componentes incluyen el conocimiento, el comportamiento y la actitud. A su vez, las acciones y el comportamiento de las personas moldean la situación financiera y su bienestar (OCDE, 2005). Además, en lo que toca a los estudiantes universitarios es determinante, toda vez que comienzan a integrarse 
a la primera fase del ciclo de vida financiero, es decir, el de acumulación de riqueza, al pasar de los ingresos negativos -dependencia - a los positivos independencia- (Garay-Anaya, 2015), de modo que la creación de una empresa es toda una posibilidad.

En razón a lo mencionado es que el objetivo de esta investigación es explorar la conducta financiera de los estudiantes universitarios de dos centros universitarios de la Universidad Autónoma del Estado de México, haciendo la distinción entre aquellos que son emprendedores, emprendedores potenciales y aquellos que no los son, con la finalidad de identificar conductas en temas de crédito, ahorro y administración del dinero, sin olvidar los niveles de inclusión en el sistema financiero formal.

Con esto se identifican dependencias entre las variables del estatus de emprendimiento (EE) con respecto al uso de productos financieros formales, la administración del dinero, el uso del crédito especialmente con las tarjetas de crédito-y la actitud hacia el dinero, entre otros. De la misma forma, existen otras relaciones importantes por destacar, sobre todo con relación a los temas de género, lugar de estudio y carrera universitaria.

Así, entonces, el artículo lo conforman, principalmente, cinco apartados. El primero presenta el marco teórico sobre el emprendedor y la conducta financiera como elemento relacionado con la alfabetización financiera. El segundo, la metodología del estudio que explica el diseño de la investigación. El tercero los resultados estadísticos obtenidos, el cuarto la discusión de los resultados en contraste con algunas otras investigaciones $y$, finalmente, el quinto las conclusiones.

\section{Marco teórico}

\subsection{Delimitación del emprendedor}

$\mathrm{E}^{\mathrm{I}}$ interés por estudiar al emprendedor nace formalmente en el siglo XVIII, cuando Cantillo lo señala como un sujeto capaz de asumir riesgos para darle dirección a una empresa (Kilby, 1971, como se cita en Carland, Hoy, Boulton y Carland, 2006; Zuluaga, 2010). Posteriormente, Schumpeter lo describe como un sujeto que transforma las sociedades gracias a sus innovaciones, pues el emprendedor es el eslabón entre la invención y la innovación, motivado por «una posición poderosa el placer de sentirse independiente y superior a los demás, el impulso de lucha y conquista constante y la satisfacción de crear» (Alonso y Fracchia, 2011, p. 6).

Bajo la normatividad mexicana, de acuerdo con el Diario Oficial de la Federación (DOF), se entenderá por emprendedores/as «las mujeres y los hombres con inquietudes empresariales, en proceso de crear, desarrollar o consolidar una micro, pequeña y mediana empresa (mipyme) a partir de una idea emprendedora» (Secretaría de Gobernación, 2013).
En este orden de ideas, el emprendedor parte desde la concepción de una idea hasta que la consolida. De la misma manera, el Global Entrepreniurship Monitor (GEM) determina una serie de etapas de emprendimiento que abarca desde la intención. De esta clasificación, y específicamente para efectos de este trabajo, se retoman dos: el emprendedor potencial y el emprendedor que se encuentra en la etapa temprana. El primero como el individuo que se percibe capaz de iniciar un negocio y que no será disuadido por miedo a fallar (Singer, Amorós y Moska, 2015). El segundo, un individuo entre 18 y 64 años perteneciente a la fase de actividad emprendedora temprana (TEA), es decir, emprendedores nacientes — cero a tres meses de operación-o propietarios de nuevos negocios - de tres meses a 3,5 años de operación(Hernández, et al., 2016; Singer et al., 2015).

Con la metodología del GEM, Hernández et al. (2016) identifican que el 69,7 \% de la población mexicana no es emprendedora. Por su parte, Canales-García, Román-Sánchez y Ovando-Aldana 
(2017), con base en la Encuesta Nacional de Ocupación y Empleo (ENOE) del primer trimestre del 2016, denotan que en México 5833 personas son emprendedoras, de las cuales únicamente 522 son jóvenes de 18 a 29 años.

\subsection{La conducta: comportamiento y actitud}

La conducta es el resultado de la suma de comportamientos y creencias, aunque muchas de estas no racionales (Tversky y Kahneman, 1985; Garay-Anaya, 2015); sin embargo, la manera de conducirnos en el mundo trae consigo consecuencias para lograr el bienestar o perderlo. En este sentido es que la conducta financiera será evaluada bajo dos elementos - pertenecientes a la de medición de la alfabetización financiera-: el comportamiento en el manejo del dinero y la actitud o creencia que se tiene sobre el uso de este.

Entre los principales organismos interesados en el tema se encuentra la Organización para la Cooperación y el Desarrollo Económicos (OCDE) que, junto con la Red Internacional de Educación Financiera (INFE, por sus siglas en inglés), han señalado el comportamiento como uno de los elementos de la alfabetización financiera, del cual se desprenden el control financiero a través del uso de presupuestos, compras planeadas y pagos a tiempo, resiliencia financiera a partir del ahorro activo y la toma de decisiones al comparar los productos. Además del comportamiento, otro elemento que determina la conducta en la actitud hacia el dinero es la visión hacia el futuro (OCDE, 2016; OCDE, 2017).

Así, los resultados del comportamiento y las creencias, en temas de finanzas, han sido de interés alrededor del mundo. Por ejemplo, en Alemania, se concluye que las personas con menor nivel de alfabetización financiera toman la pérdida de sus activos al venderlos en tiempo de crisis; es decir, solo contemplan la situación en el corto plazo (Bucher-Koenen y Ziegelmeyer, 2011); en Rusia, los más alfabetizados financieramente reportan menos disminución de gastos y más ahorro disponible en tiempos de crisis (Klapper, Lusardi y Panos, 2012); en Estados Unidos, los usuarios de 18 a 34 años de edad que usan la modalidad de pagos móviles tienden a cometer sobregiros en un $19 \%$ por encima de quienes no los utilizan (Lusardi, De Basa y Avery, 2018), entre otros. En resumen, una persona con una adecuada conducta financiera tiende al uso efectivo del dinero desde la forma como lo obtiene y lo gasta hasta como lo invierte (Kendida, Altsani y Meliany, 2017).

En este sentido, la conciencia de los comportamientos y las creencias, junto con la confianza de la aplicación de los conocimientos, desarrollan en los individuos un mejor nivel de alfabetización financiera, cuyo propósito final es el bienestar financiero (OCDE, 2017; CNBV, 2017). Mientras que el bienestar financiero, en lo conceptual, se concibe como alcance de los objetivos personales y empresariales que se verán modificados por la etapa de vida por la que atraviesa el individuo.

Así, direccionar hacia conductas financieras deseables y más racionales resulta relevante para los estudiantes universitarios cuyo estatus es el de transición hacia la primera etapa del ciclo de vida financiero (CVF), en el que el capital humano está en construcción entendido el capital humano como la acumulación de conocimientos y habilidades para ser utilizadas en su beneficio (Huston, 2012) - y el capital financiero es nulo y hasta negativo — derivado de la inversión en su capacitación-.

El estudiante, al saltar a la primera etapa, denominada de acumulación, se enfocará en la adquisición de bienes, en la conformación de una familia y la satisfacción de sus necesidades, así como en la gestión de proyectos empresariales (Zacari, 2018); por tanto, refiere a la etapa de mayor consumo y compra (GarayAnaya, 2015).

Es en esta etapa, en la que el capital humano en temas de finanzas personales debe estar fortalecido (GarayAnaya, 2015), de lo contrario seguiremos encontrado conductas no deseables tales como sobregiros de tarjetas de crédito en estudiantes universitarios (SoHyun, Grable y Bagwell , 2003; Lusardi y De Bassa, 2013), incremento en el endeudamiento por falta de administración y de elaboración de presupuestos 
(Allen y Kinchen, 2009; Kidwell, Brinberg y Turrisi, 2003), uso de financiamientos caros en jóvenes adultos y disposición de efectivo de las tarjetas de crédito (Lusardi y De Bassa, 2013), por mencionar algunas conductas erróneas.

En este orden de ideas, también cabe destacar que la familiaridad y la experiencia con los productos financieros resultan un detonante para mejorar la educación financiera. Moreno-Herrero, Salas-Velasco y Sánchéz-Campillo (2018) comprobaron esta relación en grupos del mismo nivel socioeconómico, hecho que apoyan otras investigaciones (Browna, Henchoz y Spycher, 2018).

\subsection{Contexto del ahorro, el crédito y el manejo del dinero en México}

En México se ha iniciado una etapa de seguimiento formal sobre la situación financiera individual de la población, toda vez que en el 2016 se creó la Política Nacional de Inclusión Financiera (PNIF) a cargo del Consejo Nacional de Inclusión Financiera (Conafi).

Con la información proporcionada por el Conafi a través de la ENIF 2015, a continuación, se esboza un panorama general (CNBV, 2017; CNBV 2018) en el tema de crédito, ahorro y administración del dinero, complementado por el trabajo elaborado por la OCDE (2017).

\subsubsection{Crédito y ahorro.}

El Reporte Nacional de Inclusión Financiera (RNIF) establece dos formas de crédito: el formal $y$ el informal. El primero es aquel que ofrece alguna institución financiera formalmente establecida ${ }^{5}$, mientras que el segundo incluye el prestamo de familiares, amigos, conocidos, cajas de ahorro o casas de empeño. Ahora bien, solo el $29 \%$ - 22,1 millones de adultos — tienen un crédito formal, el $38 \%$-29 millones de adultos - tienen un crédito informal, y el $46 \%$-34,7 millones de adultos- no tiene un crédito (ENIF, 2015). Las principales barreas son la falta de interés y evitar el endeudamiento, en lo que a demanda respecta; por la parte de la oferta, la tasa de interés y el incumplimiento de requisitos. En lo referente a las razones de rechazo, se destaca, en primer lugar, problemas en el buró de crédito, seguidos por la falta de comprobación de ingresos o isuficiencia, no contar con los documentos y, finalmente, no tener historial crediticio.

Cabe subrayar que existen diferencias entre el tipo de crédito - formal e informal — y la manera en que los mexicanos asignan este recurso - en sus tres primeros rubros-, toda vez que en el caso del crédito formal los individuos eligen, en primer lugar, la compra de activos - $34 \%$-, seguido del pago de comida y servicios $-22 \%-y$, posteriormente, para ser utilizado en un negocio - $22 \%$-; mientras que el crédito informal se utiliza, en un $41 \%$, con el fin de pagar comida o servicios, en un $11 \%$ en la compra de activos y solo el $6 \%$ para uso dentro de un negocio (CNBV, 2018).

Por otra parte, al ser el producto más colocado en México, la tarjeta de crédito y departamental representa un índice de morosidad del 14,92 \%; al respecto, los mexicanos manifestaron, en un $38 \%$ y un $29 \%$, haber atrasado el pago al menos una vez al año - departamental y de crédito, respectivamente-. En esta misma dirección, el $44 \%$ de la población declara pagar lo requerido a fin de no generar intereses, el 11 $\%$ paga el adeudo total, mientras que el $22 \%$ paga solo el mínimo.

Así mismo, Ponce, Seira y Zamarripa (2017) revelan que en México los tarjetahabientes asumen un costo superior al que deberían — hasta el $31 \%$ por arriba-, a causa de decisiones erróneas tales como la falta de comparación entre productos y sus costos, el uso inadecuado de diversas tarjetas a un mismo tiempo, la asignación de pagos mensuales en función de los saldos y no del costo financiero o no realizar pagos considerables en la fecha correspondiente al pago, entre otros.

El crédito formal considera tener algún crédito bancario, tarjeta de crédito bancaria, departamental, de tienda de autoservicio o con alguna otra institución financiera, tal como lo indica el RNIF. 
Al igual que el crédito, el ahorro se divide entre formal e informal. Mientras el primero toma en cuenta el uso de al menos una cuenta en el banco, el segundo se realiza por medio de cajas de ahorro en el trabajo, con amigos o conocidos, al guardar el dinero en casa, participar en tandas o compra de animales o bienes. De esta forma, el ENIF 2015 arroja que el $44 \%{ }^{6}$ de los adultos tiene un mecanismo de ahorro formal $y$, en específico, las jóvenes adultas de 18 a 30 años de edad ahorran formalmente en un $39 \%$, en tanto los de hombres de la misma edad en un $34 \%$. Aunque los porcentajes de uso de productos formales en el ahorro son superiores que en el crédito, solo el 17 $\%$ de los mexicanos manifiestan haber realizado una comparación para identificar el producto más conveniente (CNVB, 2018).

\subsubsection{Comportamiento en el manejo del dinero.}

Entre los principales resultados rescatados delENIF 2015 se encuentra que solo el $44 \%$ de la población encuestada puede afrontar una emergencia económica a partir del ahorro; el $37 \%$ lleva un presupuesto y el $76 \%$ realiza compras no planeadas. Además, existe una relación positiva entre la elaboración del presupuesto y el nivel de escolaridad, por la cual quienes tienen licenciatura lo llevan a cabo en un $58 \%$ (CNBV, 2017).

Respecto a los datos ofrecidos por la encuesta llevada a cabo por la OCDE (2017) en países del G20, se destaca que el $75 \%$ de los mexicanos encuestados indicaron que evalúan antes de realizar una compra si la pueden pagar, mientras que el $83 \%$ indica pagar sus cuentas a tiempo. Además, alrededor del $52 \%$ de los mexicanos realiza un ahorro activo, especialmente para los imprevistos. Sin embargo, también el $64 \%$ afirmó vivir al día y el $43 \%$ respondió que han tenido que pedir prestado a fin de solventar los gastos y poder concluir el mes.

Finalmente, en este reporte, y en específico en la variable de comportamiento, los hombres obtuvieron porcentajes superiores en el puntaje mínimo de la evaluación con respecto a las mujeres - 48 y $37 \%$, respectivamente-, con un nivel de significancia del 0,01 .

\section{Metodologia}

A fin de explorar la conducta financiera de los estudiantes universitarios, rescatando el comportamiento y la actitud, se aplicó un cuestionario de preguntas cerradas. La participación fue voluntaria y autoadministrada, por medio de la plataforma digital Survey Planet. El periodo de aplicación fue mayo a octubre del 2019.

Tabla 1. La característica de la muestra

\begin{tabular}{|c|c|}
\hline Variable & Estadística descriptiva \\
\hline \multirow{2}{*}{ Edad } & Media: 21,83 \\
\cline { 2 - 2 } & De: 1955 \\
\hline \multirow{2}{*}{ Género } & Hombres: $186-35,9 \%-$ \\
\cline { 2 - 2 } & Mujeres: $332-64,1 \%-$ \\
\hline \multirow{3}{*}{ Espacio universitario } & Zumpango: $293-56,6 \%-$ \\
\cline { 2 - 2 } & Ecatepec: $222-42,9 \%-$ \\
\cline { 2 - 2 } & Otro: $3-0,5 \%-$ \\
\hline
\end{tabular}

6 Cabe destacar que muchos de los mexicanos que ahorran formalmente también lo realizan de manera informal. 


\begin{tabular}{|l|c|}
\hline \multirow{4}{*}{ Carrera universitaria } & Administración: $78-15,1 \%-$ \\
\cline { 2 - 2 } & Contaduría: $133-25,7 \%-$ \\
\cline { 2 - 2 } & Derecho: $128-24,7 \%-$ \\
\cline { 2 - 2 } & Ing. en Computación e Informática: $91-17.5 \%-$ \\
\cline { 2 - 2 } & Psicología: $88-17 \%-$ \\
\hline \multirow{4}{*}{ Estatus de emprendedor } & No tienen potencial emprendedor $191-36.9 \%-$ \\
\cline { 2 - 2 } & Con potencial emprendedor: $252-48,6 \%-$ \\
\cline { 2 - 2 } & Emprendedor: $75-14,5 \%-$ \\
\hline
\end{tabular}

Fuente. Elaboración propia.

En la muestra los estudiantes pertenecen a los dos últimos periodos de la carrera universitaria. Las licenciaturas participantes fueron: Administración, Contaduría, Derecho, Ingeniería en Computación e Informática y Psicología, de dos centros universitarios de la UAEM. De 545 cuestionarios aplicados, solo se consideraron 518 , toda vez que se excluyeron aquellos que estaban fuera de los límites de tiempo -602 y 1224 segundos-, rango determinado a través de la media y la desviación estándar en el tiempo de contestación del instrumento. La descripción detallada de la muestra se identifica en la tabla 1. Es determinante mencionar que la clasificación del $\mathrm{EE}$ tiene como base la metodología del GEM, tal como se explicó en el marco teórico.
El análisis se realiza bajo la estadística descriptiva con el uso del software SPSS Statistics 9, toda vez que el alcance de esta investigación es exploratoria; para el caso se utilizan análisis de frecuencias y el uso de la prueba Chi cuadrada con el fin de encontrar asociación entre las variables, especialmente entre el EE y variables tales como la inclusión al crédito formal, el uso formal del ahorro, el comportamiento en el manejo del dinero y la actitud hacia el dinero, entre otros; se contempla un nivel de significancia por debajo de $0,05-$ p-value- .

\section{Resultados}

\subsection{Estatus del emprendedor}

Los resultados de significancia entre el EE y el género, el centro universitario y la carrera universitaria son de $0,002,0,710$ y 0,146 , respectivamente. De tal forma que la tabla 2 muestra solo la distribución de datos entre el EE y el género.

Tabla 2. EE por género

\begin{tabular}{|c|c|c|}
\hline \multirow{2}{*}{ Estatus de emprendimiento (EE) } & \multicolumn{2}{|c|}{ Frecuencia relativa } \\
\cline { 2 - 3 } & Hombre & Mujer \\
\hline Sin potencial emprendedor & 29,03 & 41,27 \\
\hline Con potencial emprendedor & 54,84 & 45,18 \\
\hline Emprendedor & 16,13 & 13,55 \\
\hline
\end{tabular}

Fuente. Elaboración propia. 


\subsection{Inclusión al crédito formal y el uso de productos formales para el ahorro}

En el tema de inclusión del crédito formal se identifica que solo 164 sujetos de la muestra -31,6 $\%$ - cuentan con algún producto formal. El tipo de crédito más mencionado son las tarjetas de crédito departamentales y bancarias, con una frecuencia de 95. Lo siguen el crédito personal y de nómina con frecuencias de 63 y 29 , respectivamente considere que los participantes seleccionaron más de un producto- - . No existe evidencia de crédito hipotecario. Los porcentajes se pueden apreciar en la figura 1.

Figura 1. Distribución del crédito formal por producto



Fuente. Elaboración propia.

El disgusto por endeudarse, la falta de necesidad y el desinterés representan las principales razones de la demanda — $45 \%$ — para no tener algún tipo de crédito formal, según declaran los estudiantes universitarios excluidos —en este ítem los estudiantes tuvieron la opción de elegir una o más opciones-, tal y como se aprecia en la figura 2.

Figura 2. Razones por las que no se obtiene un crédito formal

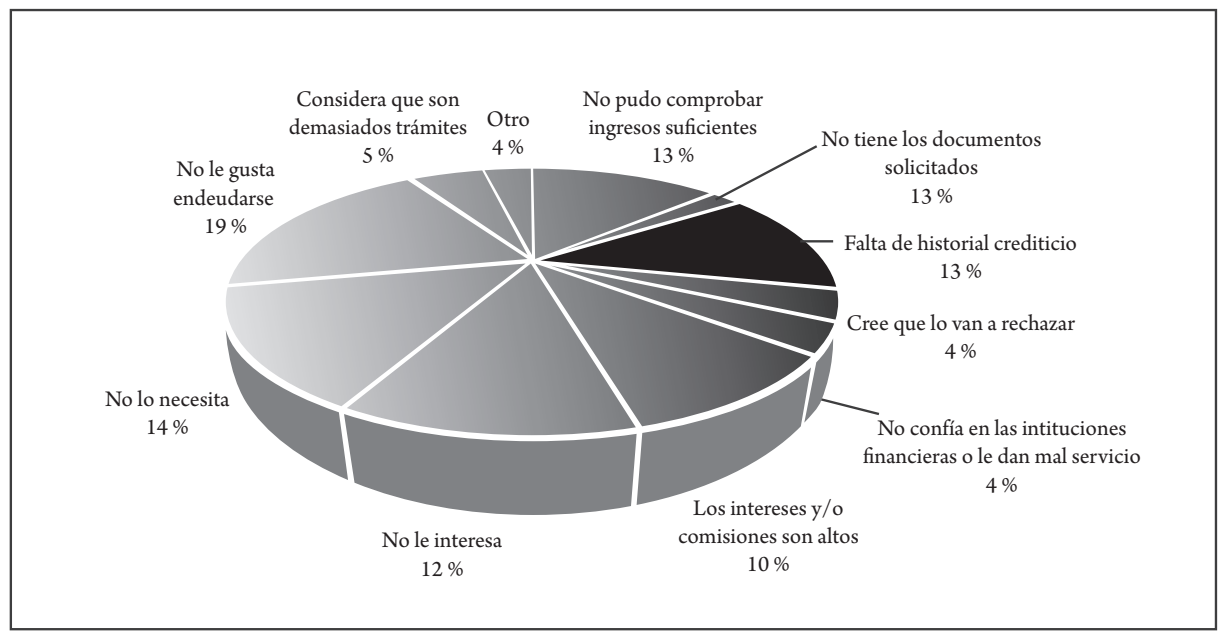

Fuente. Elaboración propia. 
Cabe subrayar que si el porcentaje de estudiantes que utilizan el crédito formal es bajo, el uso de cuentas bancarias como mecanismo de ahorro es inferior, esto es, 97 estudiantes que representan el 18,7\%, aunque 376 declararon ahorrar $-72,6 \%$ -

A continuación, se presenta la asociación entre el uso formal de productos financieros para el crédito y el ahorro con respeto al EE, el género, el espacio universitario y la carrera universitaria. El resumen se observa en la tabla 3. Entre los resultados sobresalientes se identifica que el par de variables que obtienen un p-value por debajo de 0,05 son: crédito formal y EE - 0,048-; crédito formal y carrera universitaria $-0,001-$; ahorro formal y género - 0,017-; y ahorro formal y carrera universitaria $-0,001-$.

Tabla 3. Uso formal del crédito y ahorro por estatus de emprendimiento, género, espacio universitario y carrera universitaria

\begin{tabular}{|c|c|c|c|c|c|}
\hline Variable & Categoría & $\begin{array}{l}\text { Crédito formal } \\
\text { Frecuencia relativa } \\
(\%)\end{array}$ & p-value & $\begin{array}{l}\text { Ahorro formal } \\
\text { Frecuencia relativa } \\
(\%)\end{array}$ & p-value \\
\hline \multirow{3}{*}{$\mathrm{EE}$} & Sin potencial emprendedor & 26,18 & \multirow{3}{*}{0,408} & 17,80 & \multirow{3}{*}{0,903} \\
\hline & Con potencial emprendedor & 32,94 & & 19,05 & \\
\hline & Emprendedor & 41,33 & & 20,00 & \\
\hline \multirow{2}{*}{ Género } & Masculino & 33,87 & \multirow{2}{*}{0,048} & 24,19 & \multirow{2}{*}{0,017} \\
\hline & Femenino & 30,42 & & 15,66 & \\
\hline \multirow{2}{*}{$\begin{array}{c}\text { Espacio } \\
\text { universitario }\end{array}$} & Zumpango & 31,06 & \multirow{2}{*}{0,737} & 17,75 & \multirow{2}{*}{0,515} \\
\hline & Ecatepec & 32,44 & & 20,00 & \\
\hline \multirow{5}{*}{$\begin{array}{c}\text { Carrera } \\
\text { universitaria }\end{array}$} & Administración & 47,44 & \multirow{5}{*}{0,001} & 20,51 & \multirow{5}{*}{0,001} \\
\hline & Contaduría & 34,59 & & 30,08 & \\
\hline & Derecho & 32,81 & & 16,41 & \\
\hline & $\begin{array}{l}\text { Ing. en Computación e } \\
\text { Informática }\end{array}$ & 23,08 & & 13,19 & \\
\hline & Psicología & 20,45 & & 9,09 & \\
\hline
\end{tabular}

Fuente. Elaboración propia.

Observe cómo el estudiante emprendedor tiene mayor acceso al crédito con un 41,33\%, y cómo las carreras dentro del área económica administrativa tienen una mayor participación en el uso de los productos financieros formales.

\subsection{Comportamiento en el manejo de tarjetas de crédito}

Una vez mencionados los principales resultados relacionados con la inclusión, se procede a describir el comportamiento con respecto al uso de las tarjetas de crédito, especialmente resaltando las diferencias significativas entre los grupos del EE. En este sentido, 81 sujetos declararon tener al menos una tarjeta bancaria o departamental: $52-64,2 \%$ - con tarjeta departamental, 15 con tarjeta bancaria - 18,5\%-y 14 estudiantes con ambas tarjetas - 17,3\%-. En lo referente a la distribución de tarjeta de crédito por EE, la tabla 4 muestra cómo el grupo emprendedor tiene, en mayor porcentaje relativo, una tarjeta de crédito. 
Tabla 4. Tarjeta de crédito por EE

\begin{tabular}{|c|c|c|c|}
\hline Estatus emprendedor & Con tarjeta de crédito & Total por grupo & Frecuencia relativa -\%- \\
\hline Sin potencial emprendedor & 29 & 191 & 15,18 \\
\hline Con potencial emprendedor & 34 & 252 & 13,49 \\
\hline Emprendedor & 18 & 75 & 24,00 \\
\hline Total & 81 & 518 & \\
\hline
\end{tabular}

Fuente. Elaboración propia.

Ahora bien, la significancia entre el EE y el pago a tiempo de la tarjeta de crédito fue de 0,182; sin embargo, el nivel de significancia entre el $\mathrm{EE}$ y la forma de pago fue de 0,000. En la figura 3 se visualiza que el estudiante sin potencial y el estudiante con potencial pagan la cantidad requerida para no generar intereses, mientras que el emprendedor paga el total de la deuda en mayor proporción.

Figura 3. Conducta en la forma de pago de la tarjeta de crédito por estatus de emprendimiento

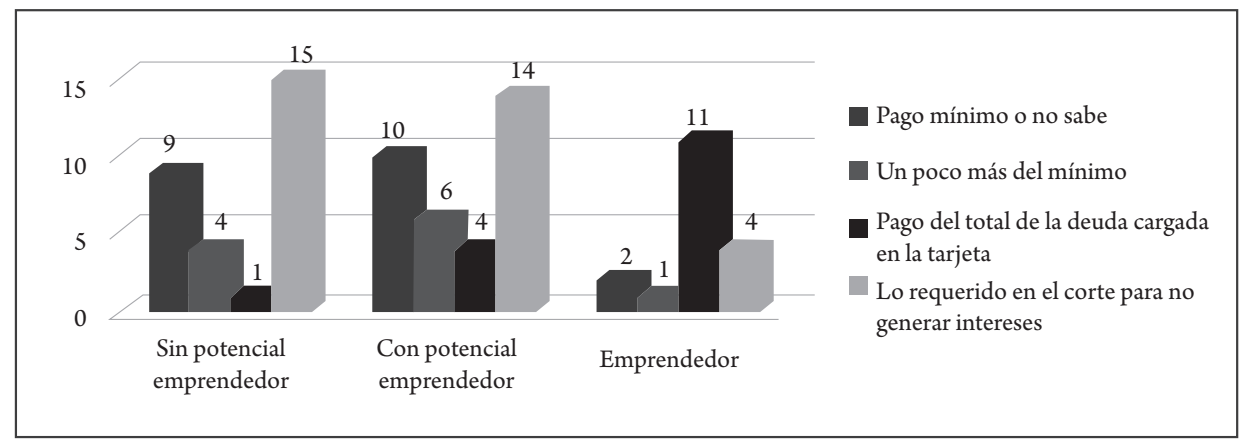

Fuente. Elaboración propia.

\subsection{Comportamiento en el manejo del dinero}

En términos generales, el 53,1\% de los estudiantes presupuestan los ingresos y egresos, el 71,4 \% lleva a cabo acciones para disminuir gastos innecesarios, el $58,7 \%$ no realiza compras de manera impulsiva, el $47,1 \%$ ha realizado un análisis de su gasto hormiga y el $58,1 \%$ lleva registro de sus gastos.
¿Cuál es la relación entre el comportamiento y el $\mathrm{EE}$, el género, el lugar de adscripción universitaria y la carrera? Los resultados se observan en la tabla 5; sin embargo, no todas las asociaciones mantienen significancias.

Tabla 5. Nivel de significancia entre comportamientos y estatus del emprendedor, género, centro universitario y carrera universitaria

\begin{tabular}{|l|c|c|c|c|}
\hline Comportamiento & EE & Género & CU & Carrera universitaria \\
\hline Pago de cuentas a tiempo & 0,002 & 0,556 & 0,601 & 0,203 \\
\hline Elaboración de un presupuesto & 0,091 & 0,749 & 0,133 & 0,000 \\
\hline Acciones para disminuir gastos innecesarios & 0,638 & 0,977 & 0,001 & 0,938 \\
\hline Compras de manera impulsiva & 0,856 & 0,067 & 0,152 & 0,590 \\
\hline Análisis sobre el gasto hormiga & 0,019 & 0,059 & 0,099 & 0,000 \\
\hline Registro de gastos & 0,013 & 0,449 & 0,754 & 0,063 \\
\hline Contar con un fondo para atender imprevisto & 0,070 & 0,001 & 0,000 & 0,017 \\
\hline
\end{tabular}

Fuente. Elaboración propia. 
El EE tiene una asociación con el pago de las cuentas a tiempo - $p$-value 0,002_, el análisis del gasto hormiga - $p$-value $0,019-$, y el registro de los gastos $-p$-value 0,013 - El emprendedor obtiene mayor porcentaje en el análisis del gasto hormiga —62,67\%—y el registro de los gastos — 56,00\%—, mientras que el emprendedor potencial obtiene el mayor porcentaje en el pago de cuentas a tiempo - 65,56 \%-, datos presentados en la figura 4.

Figura 4. Conducta en relación con el estatus de emprendimiento — frecuencias relativas-



Fuente. Elaboración propia.

Por parte de la variable de género, únicamente guarda una dependencia con el fondo para atender un gasto imprevisto — $p$-value-0,001—. Así, el 47,9\% de las mujeres tienen un fondo de emergencia para imprevistos en contraste con el $32,4 \%$ de los hombres.

Por espacio universitario, la relación se demuestra con las acciones dirigidas a disminuir el gasto $-p$-value $0,001-y$ contar con un fondo de ahorro para un gasto imprevisto $-p$-value $0,000-$. De este último, el 50,3\% de los estudiantes de CU Zumpago cuenta con el fondo de ahorro para imprevistos, mientras los del CU Ecatepec solo en un 31,8 \%. De la misma forma, CU Zumpango obtuvo un mayor porcentaje con respecto a la disminución de gastos con el 77,1\%, en contraste con el 64,0 \% de CU Ecatepec.

La elaboración del presupuesto $-p$-value $0,000-$, el análisis y el gasto hormiga — $p$-value $0,000-$, así como contar con un fondo de ahorro para algún imprevisto $-p$-value 0,017 - se relacionó con la carrera universitaria. En la figura 5 se puede observar que las prácticas de llevar a cabo el análisis y la planeación se encuentran en la carrera de Contaduría -63,9 \% y 68,42 \%-. Sin embargo, Psicología tiene mayor incidencia en tener un fondo de ahorro para los imprevistos. 
Figura 5. Conducta en relación con la carrera universitaria — frecuencias relativas-

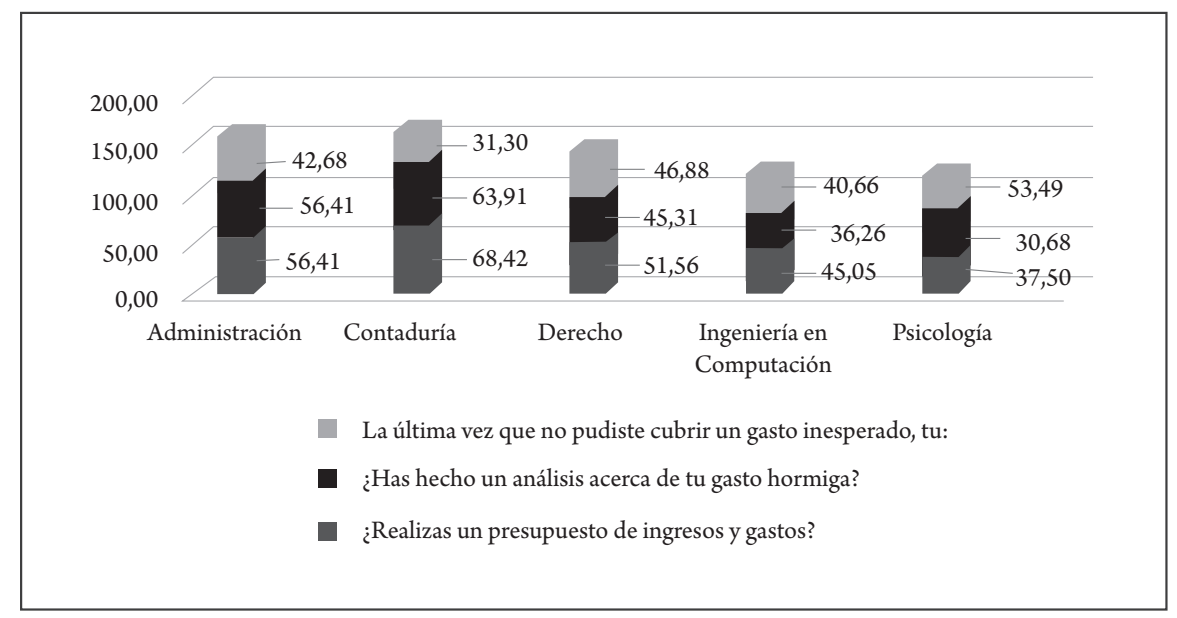

Fuente. Elaboración propia.

\subsection{Actitud hacia el dinero y el futuro}

El 64,5\% de los estudiantes universitarios están en desacuerdo con encontrar mayor satisfacción en gastar el dinero que ahorrarlo por un largo tiempo. No se encontró niveles de significancia entre la actitud hacia el dinero y el EE, el género, el CU y la carrera universitaria; los resultados de p-value respectivos son: $0,399,0,566,0,394$ y 0,075 .

¿Cuál es la actitud con respecto al uso del crédito por EE? Más de la mitad de la muestra manifestó interés por generar un patrimonio, ya sea empresarial —32,8 $\%$ - o personal - $24,71 \%$ - Los datos arrojados indican que el 52,00 \% de los emprendedores utilizarían el crédito para ampliar o iniciar algún negocio, mientras que los emprendedores potenciales alcanzaron el 40,5\%; finalmente, el estudiante sin potencial emprendedor lo declara en un $30 \%$. La actitud es a la inversa con respecto al patrimonio personal, como, por ejemplo, la construcción o la ampliación de una casa o la compra de algún bien duradero. Es decir, en primer lugar el estudiante sin potencial emprendedor, seguido del emprendedor potencial $y$, finalmente el emprendedor, con los siguientes porcentajes respectivos: $26,3 \%, 24,2 \%$ y $22,7 \%$. Cabe destacar que esta asociación de variables alcanzó un 0,001 de p-value.

\section{Discusión}

$\mathrm{T}$ al y como se ha planteado en la parte inicial de este artículo, la actividad emprendedora resulta trascendental en el crecimiento de las economías por sus múltiples beneficios, de tal forma que la participación de la población más preparada es decisiva para la generación innovadora de modelos de negocios. Así, entonces, una vez analizada la información, se identifica que el 85,5 \% de los estudiantes participantes no son emprendedores, en contraste con el 69,7
\% que determina el GEM (Hernández, et al., 2016); sin embargo, existe un porcentaje representativo en el emprendedor potencial, lo que se traduce en una oportunidad, toda vez que el promedio de edad de esta muestra fue de 21,83 años. En este sentido, se ha encontrado evidencia de relación positiva entre la actividad emprendedora y la edad en jóvenes entre los 18 y los 29 años (Canales-García et al., 2017). 
Otro dato relevante es que los hombres, estudiantes universitarios, son más propensos a participar en actividades emprendedoras, ya que tienen mayor presencia en los grupos de emprendimiento potencial y emprendedor. Resultado por género que se confirma en el estudio realizado por Canales-García et al. (2017) con los datos de la ENOE y en los resultados del GEM (Hernández et al., 2016).

Ahora bien, también en el inicio de este documento se explica que una de las principales barreras del ecosistema emprendedor es la falta del financiamiento (GEM, 2015; 2017). En este caso, el estudiante universitario tiene mayor acceso a algún tipo de crédito formal, en comparación con los resultados arrojados por el ENIF 2015, con 2,6 puntos porcentuales de diferencia. Aunque cabe destacar que las razones por las cuales los estudiantes no utilizan el crédito formal se relacionan más con una barrera de demanda que de oferta, es decir, por disgusto a endeudarse, desinterés o falta de necesidad.

En lo que corresponde al ahorro, los estudiantes manifestaron en un alto porcentaje llevar a cabo, sin embargo, solo el 18,7 \% declaró utilizar alguna cuenta bancaria para realizarlo, muy por debajo de la media nacional que reporta el ENIF 2015, aun en la población joven.

¿Cuál es la asociación del crédito y el ahorro formal con las otras variables? Se identificó que existe una asociación entre el crédito formal y el EE. De esta asociación se desprende que el emprendedor tiene un mayor acceso al crédito formal con respecto al emprendedor potencial, y este, a su vez, al que no tiene potencial emprendedor. Además, el emprendedor presenta un mayor acceso a las tarjetas de crédito, de las cuales la más utilizada es la departamental. Este dato coincide con los de la ENIF 2015, pues subraya las tarjetas de crédito como las más ofertadas por la banca. En el tema de forma de pago de tarjetas, los emprendedores potenciales seleccionaron como principal respuesta pagar el monto requerido a fin de no generar intereses en el periodo, mientras que el emprendedor tiende a pagar el total de la deuda cargada en la tarjeta.
Así mismo, el crédito y el ahorro formal están asociados con la carrera universitaria, pues en ambas las carreras del área económica-administrativa tienen mayor participación. De la misma forma, se identifica asociación entre el ahorro formal y el género, encontrando que los hombres utilizan más productos financieros formales para llevar a cabo el ahorro. Sherraden, Peters, Wagner, Guo y Clancy (2013) identifican que el uso de cuentas formales motiva y facilita el ahorro en niños y jóvenes.

Siguiendo con la discusión, se determinó analizar la conducta financiera de los estudiantes a partir de dos elementos de la alfabetización financiera: el comportamiento y la actitud, toda vez que la alfabetización financiera permite a las personas conducirse y enfrentar los retos económicos personales y las contingencias (Lusardi, Scheneider y Turano, 2011). En esta dirección, se observó en el comportamiento de los estudiantes que más del 50 $\%$ de ellos lleva a cabo las prácticas para mejorar la planeación y el control del dinero, a excepción de la práctica del análisis del gasto hormiga.

Con dependencia entre EE y comportamientos, en el manejo del dinero se detecta que el estudiante sin potencial emprendedor se comporta de manera menos deseable en el pago a tiempo de las cuentas, el análisis hormiga y el registro de los datos, en contraste con el emprendedor, quien lo realiza en mayor porcentaje. Esto podría resultar racional, pues quien tiene un patrimonio o invierte tiende a preocuparse más por mejorar su conocimiento financiero, «ya que tienen más en juego» (Lusardi y Michell, 2011). En este sentido un emprendedor compromete parte de su patrimonio, y su satisfacción financiera se relaciona con su conciencia y comportamiento financiero, pues de manera adecuada el emprendedor percibe un mejor control de su condición financiera en el futuro (Kendida et al., 2017).

Por género y comportamiento — con significancia-, se identifica que las mujeres tienden más a tener un fondo para los imprevistos. Aunque, según la OCDE (2017), el hombre tiende más a comportamientos que mejoran su bienestar financiero. 
La prevención, por su parte, al igual que las acciones para disminuir gastos, se presenta más en estudiantes del CU Zumpango, quienes tienden a realizar acciones con miras a disminuir los gastos innecesarios. Además, por carrera se identifica que las carreras relacionadas con el área económico-administrativo practican más el análisis y la planeación del dinero, aunque quienes prevén el ahorro para imprevistos son estudiantes que provienen de la carrera de psicología.

Finalmente, con respecto a la actitud hacia el dinero, más de la mitad manifestó que no le resulta más satisfactorio gastar el dinero hoy que en el futuro
-64,5\%-, porcentaje superior al presentado por la ODCE (2017), que en el caso de México es del 49,0 $\%$. Así mismo, los estudiantes, en el caso hipotético de contar con los recursos los utilizarían, en primer lugar, para generar un patrimonio empresarial iniciar o ampliar una empresa- con el 38,2\%, seguido del patrimonio personal con el 24,71 \% compra de casa o bien duradero-. Aunque entre grupos se identifica que el más interesado en el patrimonio empresarial es el emprendedor, mientras que en el patrimonio personal es de mayor interés para el estudiante sin emprendimiento potencial.

\section{Conclusiones}

La información descrita a lo largo de este documento, derivado de una investigación exploratoria, resulta valiosa como evidencia empírica sobre la conducta financiera de los estudiantes universitarios. En especial, como prueba de que existe una relación entre la conducta financiera y el emprendimiento.

A pesar de que los emprendedores manifiestan, en mayor medida, comportamientos que elevan su bienestar financiero, se requiere mejorar, pues con lo ya listado existe un sinfín de factores que no benefician al perfil del estudiante universitario, y la estadística no dista - y en otros casos no mejoracomo en algunos resultados de la ENIF y de la OCDE. Sin embargo, cabe subrayar que para generalizar resultados es necesario replicar el estudio en otros espacios, tanto de la misma universidad como de otras IES. Lo anterior brinda la oportunidad de contrastar resultados $y$, posteriormente, identificar variables que tengan mayor incidencia en el comportamiento financiero de aquellos que inician su ciclo.

Por lo pronto, los espacios académicos estudiados, especialmente elárea de fomento de cultura empresarial de dichas unidades, cuentan con elementos iniciales para activar acciones que promuevan conductas financieras más sanas y sostenibles entre su comunidad estudiantil.
Finalmente, a partir de los resultados nace el interés por analizar grupos específicos, tal como es el caso de los estudiantes de psicología, quienes obtuvieron menor incidencia en comportamiento financiero deseable - a excepción de la generación de un fondo de ahorroo el de los emprendedores universitarios en el uso de determinados productos financieros.

\section{Referencias}

Allen, K.; Kinchen, V. (2009). Financial management practices of college students. Global Journal of Business Reseach, 3(1), 105116.

Alonso, C.; Fracchia, E. (2011). El emprendedor schumpeteriano: aportes a la teoría económica moderna. Revista de Administración, Finanzas y Economía, 5(1), 1-22.

Atkinson, A. (2017). Financial education for MSMEs and potential entrepreneurse. OECD Working Papers on Finance, Insurance and Private Pensions (43). DOI: http://doi. org $/ 10.1787 / \mathrm{bb} 2 \mathrm{~cd} 70 \mathrm{c}$-en

Browna, M.; Henchoz, C.; Spycher, T. (2018). Culture and financial literacy: evidence from a within-country. Journal of Economic Behavior \& Organization, (150), 62-85. DOI: https://doi.org/10.1016/j.jebo.2018.03.011

Bucher-Koenen, T.; Ziegelmeyer, M. (2011). Who Lost the Most? Financial Literacy, Cognitive Abilities, and the Financial Crisis. MEA BCL working papers, Central Bank of Luxembourg Discussion, 234(11), 1-29. DOI: http://dx.doi.org/10.2139/ ssrn. 1738368 
Canales-García, R. A.; Román-Sánchez, Y. G.; Ovando-Aldana, W. (2017). Emprendimiento de la población joven en México. Una perspectiva crítica. Entreciencias: Diálogos en la Sociedad del Conocimiento, 5(12), 1-17. DOI: https://doi. org/10.21933/J.EDSC.2017.12.211

Carland, J W.; Hoy F., Boulton W. R.; Carland J. A. C. (2006). Diferenciar a los emprendedores de los dueños de pequeñas empresas: una conceptualización. Entrepreneurship: concepto, teoría y perspectiva. España: Fundación Banjaca.

CNBV (Comisión Nacional Bancaria y de Valores). (2017). Encuesta Nacional de Inclusión Financiera. Recuperado de https://bit.ly/3cC311A

CNBV (Comisión Nacional Bancaria y de Valores). (2018). Reporte Nacional de Inclusión Financiera 9. Recuperado de https://bit.ly/2ODkZvW

Conaif (Consejo Nacional de Inclusión Financiera). (2016). Política Nacional o Inclusión Financiera. Recuperado de https://bit.ly/3rUiV10

Garay-Anaya, G. (2015). Las finanzas conductuales, el alfabetismo financiero y su impacto en la toma de decisiones financieras, el bienestar económico y la felicidad. Perspectivas, 35, 7-34. Recuperado de https://bit.ly/30RQeps

GEM (Global Entrepreneurship Monitor). (2013). Monitor Global de la Actividad Emprendedora México 2013. Recuperado de https://bit.ly/3s6klW4

GEM (Global Entrepreneurship Monitor). (2015). Reporte regional 2015, México. Recuperado de https://bit.ly/3tqAYfx

GEM (Global Entrepreneurship Monitor). (2017). Global Report 2016/17. Recuperado de https://bit.ly/3rVrAjB

Hernández, K.; González, L.; Herrera, M. (2016). Reporte Regional 2015, México. México: ITESM-GEM. Recuperado de https://bit.ly/2NnfEbp

Huston, S. (2012). Assessing financial literacy. En D. Durband; S. Britt (Eds.) Student financial literacy. (109-124). Boston, MA: Springer. DOI: https://doi.org/10.1007/978-1-4614-35051_9

IMCO (Instituto Mexicano para la Competitividad). (2014). Los emprendedores de TIC en México. Recomendaciones de política pública para su nacimiento, crecimiento y consolidación. Recuperado de https://bit.ly/3s6kB7u

Kendida, B.; Altsani, W.; Meliany, Y. (2017). Financial literacy and financial behavior as mesuare of financial satisfaction.1st Economics and Business International Conference 2017. DOI: https://doi.org/10.2991/ebic-17.2018.79

Kidwell, B.; Brinberg, D.; Turrisi, R. (2003). Determinants of money management behavior. Journal of Applied Social Psychology, 33(6), 1244-1260. DOI: https://doi. org/10.1111/j.1559-1816.2003.tb01948.x
Klapper, L. F.; Lusardi, A.; Panos G. A. (2012). Financial literacy and the financial crisis (Working Paper 17930). National Bureau of Economic Research. DOI: https://doi. org/10.1596/1813-9450-5980

Lupiáñez C. L.; Priede B. T.; López-Cózar N. C. (2014). El emprendimiento como motor del crecimiento económico. Boletín económico de ICE, (3048), 55-63.

Lusardi, A.; Bassa S. C. de. (2013) Financial literacy and high-cost borrowing in the United States (NBER Working Paper, núm. 18969). DOI: https://doi.org/10.2139/ssrn.2585243

Lusardi, A.; Bassa S. C. de; Avery, M. (2018). Millennial mobile payment users: a look into their personal finances and financial behaviors. Recuperado de https://bit.ly/3cAFT72

Lusardi, A.; Mitchell, O. (2016). La importancia económica de la alfabetización financiera: teorías y pruebas. Boletín CEMLA, 301-248. Recuperado de https://bit.ly/3eKAMDO

Lusardi, A.; Mitchell, O. S. (2011). Financial literacy and planning: implications for retirement wellbeing (working paper núm. 17078). DOI: https://doi.org/10.3386/w17078

Lussardi, A.; Scheneider, D.; Tufano, P. (2011) Financial fragile household: Evidence and implizatons. NBER Working Paper, núm 17072. Recuperado de https://bit.ly/3eKWLul

Messina M.; Pena J. (2016). Indicadores de la educación emprendedora en Uruguay. Proyecto IEEL. Recuperado de https://bit.ly/2OXgVq7

Moreno-Herrero, D.; Salas-Velasco, M.; Sánchez-Campillo, J. (2018). Factors that influence the level of financial literacy among young people. Children and Youth Services Review, (95), 334-351. DOI: https://doi.org/10.1016/j. childyouth.2018.10.042

OCDE (Organisation for Economic Co-operation and Development). (2016). OECD/INFE International Survey of Adult Financial Literacy Competencies. Recuperado de https:// bit.ly/3vvW9ie

OCDE (Organisation for Economic Co-operation and Development). (2017). G20/OECD INFE report on adult financial literacy in G20 countries. Recuperado de https://bit. ly/3llaedA

OECD (Organisation for Economic Co-operation and Development). (2005). Recommendation on principles and good practices for financial education and awareness. Recuperado de https://bit.ly/2Qfbi7z

Olaya E. S.; Berbegal-Mirabent, J.; Duarte O. G. (2014). Desempeño de las oficinas de transferencia universitarias como intermediarias para la potencialización del mercado de conocimiento. Intangible Capital, 10(1), 155-188. DOI: https://doi.org/10.3926/ic.497 
Ponce, A.; Seira, E.; Zamarripa, G. (2017). Borrowing on the wrong credit card? Evidence from Mexico. American Economic Review, 107(4), 1335-1361. DOI: https://doi.org/10.1257/ aer.20120273

Salinas-Ramos F.; Osorio-Bayter L (2012). Emprendimiento y economía social, oportunidades y efectos en una sociedad en transformación. Ciriec-España, Revista de Economía Pública, Social y Cooperativa, (75) 129-151.

Secretaría de Gobernación. (15 de abril del 2013). Acuerdo que regula la organización y funcionamiento interno del Instituto Nacional del Emprendedor. Diario Oficial de la Federación DOF. Recuperado de https://bit.ly/3rTfLur

Sherraden, M.; Peters, C.; Wagner, K.; Guo, B.; Clancy, M. (2013). Contributions of qualitative research to understanding savings for children and youth. Economics of Education Review, 32, 66-77. DOI: https://doi.org/https://doi.org/10.1016/j. econedurev.2012.09.006

Singer, S.; Amorós, J.; Daniel, M. (2015). Global Entrepreneurship Monitor 2014. Global Report. GEM.
So-Hyun, J.; Grable J. E.; Bagwell C. (2003). Credit card attitudes and behaviors of college students. College Student Journal, 37(3), 405-419. Recuperado de https://bit.ly/3qSbN3X

Sparano R. H. (2014). Emprendimiento en América Latina y su impacto en la gestión de proyectos. Revista Dimensión Empresarial, (12), 95-106. DOI: https://doi.org/10.15665/ rde.v12i2.281

Tversky, A.; Kahneman, D. (1985). The framing of decisions and the psychology of choice. Science, 2011(4481), 452-458. DOI: https://doi.org/10.1126/science.7455683

Zacari, A. (2018). Finanzas personales y ciclo de vida. Invenio Universidad del Centro Educativo Latinoamericano, 11(020), 63-71. Recuperado de https://bit.ly/3qTPcEf

Zuluaga J. C. (2010). Emprendimiento e historia empresarial. Apuntes para un diálogo interdisciplinario en América Latina. Memoria del Congreso Latinoamericano de Historia Económica. Sesión II. IV Congreso Internacional de la Asociación Mexicana de Historia Económica, México D. F. 